\title{
Six tips for your (systematic) literature review in business and management research
}

\author{
Christian Fisch $^{1,2}$. Joern Block ${ }^{1,2}$
}

Published online: 3 May 2018

(C) Springer International Publishing AG, part of Springer Nature 2018

With the start of our editorial term, we decided to extend Management Review Quarterly's (MRQ's) scope beyond (systematic) literature reviews ${ }^{1}$ to include also bibliographic studies, meta-analyses, and replication studies. Nevertheless, literature reviews have been and will continue to be a core element of MRQ. Literature reviews have given the journal a unique identity and are crucial in the pursuit of the journal's aim, which is to summarize, categorize, and challenge existing knowledge in business and management research. In this editorial, we outline six tips that help (MRQ) authors to improve their literature review.

A literature review is an essential component of almost any research project. It serves as the foundation for advancing knowledge, facilitates theory development, closes mature research areas, and uncovers novel research areas (Webster and Watson 2002). Frank and Hatak (2014) refer to a literature review as a "knowledge map", which analyzes and synthesizes prior literature. Because literature reviews are so prevalent, there exist already several comprehensive resources that guide authors through the steps necessary to conduct a literature review (e.g., Aguinis et al. 2018; Booth et al. 2016; Frank and Hatak 2014; Tranfield et al. 2003; Webster and Watson 2002).

\footnotetext{
${ }^{1}$ Note that the term "systematic literature review" is not clearly defined. In MRQ's understanding, it refers to all literature reviews that follow a systematic, transparent, and reproducible process for identifying academic literature about a clearly defined topic or research question.
}

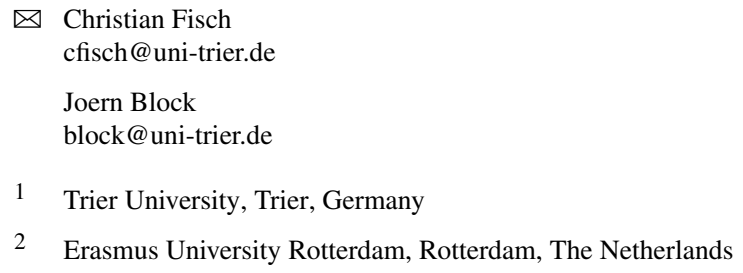


Surprisingly, there is a still considerable variance in the understanding of what a literature review is and, consequently, in the quality of systematic literature reviews. Often, researchers seem unfamiliar with the process, structure, and presentation of systematic literature reviews and produce merely descriptive, annotated bibliographies of loosely connected research, which makes it unnecessary complex and difficult for the readers to follow the literature review. The literature review therefore does not achieve its main goal of summarizing and categorizing knowledge.

There is also the misconception that literature reviews are less rigorous or easier to write than empirical articles. However, conducting a literature review of high quality requires an in-depth understanding of the necessary processes and skills and is by no means a trivial endeavor. It also requires some experience in the respective field, as the interpretation of the results of the studies included in the literature review is subjective and by no means trivial.

Here, we outline six suggestions that we think are crucial for every literature review:

1. Motivate the topic and state the research question The abstract and introduction are crucial elements of any research article. Usually, the reader decides after looking at the abstract and/or introduction whether he will read the entire article or not. Additionally, a literature review needs a crisp and concise motivation. It is important to not only motivate why a topic warrants investigation but also why the authors choose to approach the topic in the form of a (systematic) literature review. Perhaps the most important element of an introduction is the research question that guides the remainder of the literature review. Therefore, we encourage authors to carefully develop and clearly state their research question(s) in the introductory section.

2. Identify the relevant literature in a systematic way A distinguishing feature of a systematic literature review is that the review process should be transparent and reproducible. The authors need to clearly outline their search strategy for identifying relevant literature in a systematic way to establish as much transparency as possible. This involves a description of the databases where the literature search was conducted, a definition of the search terms and keywords used to identify literature, and a careful description of the practical (e.g., language, availability) and methodological (e.g., time frame, article type) screening and exclusion criteria used. Notice that the application of screening criteria (e.g., only focusing on highly ranked journals) should be well-justified, as screening criteria can have crucial implications for the results and their generalizability.

3. Choose the right balance between breadth and depth When conducting a systematic literature review, authors often face the dilemma of choosing between breadth and depth when identifying and describing prior studies. In general, a good systematic literature review is characterized by the right balance between breadth and depth by including all relevant studies but only describing important studies in more detail in a structured way. This dilemma is often difficult to solve, as a literature review should be coherent and cover a research field as a whole, but it should not be an endless, overly descriptive summary of all studies that the authors identified. To solve this dilemma to some extent, authors should make use of tables and figures to convey the most important concepts and information in an efficient 
fashion. For example, figures can be used to illustrate the development of the number of studies over time and can also illustrate which topics have attracted the most research. Of course, tables and figures should be used in a sensible fashion and should never present the main focus of the literature review. The breadth and depth of a literature review also depend on the maturity of the research field. A literature review on a mature topic requires that the authors analyze and synthesize a large body of literature, in comparison to a review on a more novel field where only few studies exist. The authors of this editorial were once challenged to summarize the literature on the intersection of entrepreneurship and innovation. At first, this seemed to be an impossible task. However, after having developed a more concise research question (see suggestion 1), developing clear inclusion and exclusion criteria (see suggestion 2), focusing on breadth instead of depth (see suggestion 3 ) and on concepts rather than studies (see suggestion 4), the task became doable. The whole process took many iterations and was very work-intensive, but we managed to deliver a literature review at the intersection of innovation and entrepreneurship or vice versa (see Block et al. 2017).

4. Focus on concepts, not studies Authors need to decide how to summarize and categorize the literature identified. While it is possible to summarize literature in a chronological or even alphabetical order, we believe that literature reviews should be concept-centric. This involves a careful identification and evaluation of the underlying concepts used in the review, which then guide the analysis conducted. Focusing on concepts instead of studies helps authors to identify the research debates they aim to contribute to and helps to ensure a better structure throughout the manuscript. Hence, a systematic literature review needs to be based on sound logical and conceptual reasoning. This can (but need not) lead to a new conceptual framework with propositions. In this sense, writing a systematic literature review very much resembles the writing of a conceptual theory paper.

5. Derive meaningful conclusions Closely connected to the previous point, we want to reiterate that a systematic literature review must go beyond a mere descriptive summary of prior literature. While it is important to provide a descriptive overview on the topics and studies included, it is essential to go one step further and to synthesize and interpret this knowledge. The literature review should derive meaningful conclusions and needs to answer the question: What do we learn from this summary? This includes carefully evaluating and deriving implications, pointing out gaps in the literature, and outlining avenues for future research.

6. Follow a coherent article structure A coherent structure is a crucial element of any research article. The structure of a systematic literature review resembles the structure of an empirical article. The introduction motivates the topic and describes the contributions of the literature review. The next section describes the systematic review process and the key concepts used. After that, the crucial part is the synthesis and interpretation of the literature review's findings. This section can but need not lead to the derivation of propositions or a conceptual model (see suggestion 4 above). The final section of a literature review provides a conclusion and discussion with the boundaries of the review and the future research areas. The order of the sections is not static and can vary depending on the review's topic. For example, one can also put suggestions for future research directly into the body of 
the article where the main findings from the literature review are described and/or discussed. However, a coherent structure is an absolute necessity for a systematic literature review.

In addition to incorporating these six suggestions, we encourage authors interested in submitting a systematic literature review to MRQ to carefully read the references provided in this article.

Acknowledgements We thank Andreas Kuckertz (University of Hohenheim) and Alexandra Moritz (Trier University) for their valuable comments that greatly improved the manuscript.

\section{References}

Aguinis H, Ramani RS, Alabduljader N (2018) What you see is what you get? Enhancing methodological transparency in management research. Acad Manag Ann 12(1):83-110

Block J, Fisch C, van Praag M (2017) The Schumpeterian entrepreneur: a review of the empirical evidence on the antecedents, behavior, and consequences on innovative entrepreneurship. Ind Innov 24(1):61-95

Booth A, Sutton A, Papaioannou D (2016) Systematic approaches to a successful literature review. Sage, Thousand Oaks

Frank H, Hatak I (2014) Doing a research literature review. In: Fayolle A, Wright M (eds) How to get published in the best entrepreneurship journals. Edward Elgar, Cheltenham, pp 94-117

Tranfield D, Denyer D, Smart P (2003) Towards a methodology for developing evidence-informed management knowledge by means of systematic review. Br J Manag 14(3):207-222

Webster J, Watson RT (2002) Analyzing the past to prepare for the future: writing a literature review. MIS Q 26(2):xiii-xxiii 